\title{
Téoros
}

Revue de recherche en tourisme

\section{Le concept de destination}

Diversité sémantique et réalité organisationnelle

\section{Boualem Kadri, Mohamed Reda Khomsi et Maria Bondarenko}

Volume 30, numéro 1, 2011

L'excellence des destinations

URI : https://id.erudit.org/iderudit/1012104ar

DOI : https://doi.org/10.7202/1012104ar

Aller au sommaire du numéro

\section{Éditeur(s)}

Université du Québec à Montréal

ISSN

0712-8657 (imprimé)

1923-2705 (numérique)

Découvrir la revue

Citer cet article

Kadri, B., Khomsi, M. R. \& Bondarenko, M. (2011). Le concept de destination : diversité sémantique et réalité organisationnelle. Téoros, 30(1), 12-24.

https://doi.org/10.7202/1012104ar

\section{Résumé de l'article}

Le concept de destination fait l'objet d'une grande utilisation en tourisme, tant dans le monde professionnel que dans celui de la communauté scientifique. Cette situation montre toutefois un paradoxe : si l'on reconnaît la puissance du mot destination relative notamment à sa richesse anthropologique, l'on observera néanmoins un déficit définitionnel, voire l'absence d'une définition partagée par la communauté scientifique. À quelles significations renvoie le mot destination ? Le présent article expose les résultats d'une analyse sémantique et conceptuelle du concept de destination réalisée à partir d'un corpus d'écrits portant plus particulièrement sur le tourisme urbain. À la lumière de cette analyse, une nouvelle perspective d'appréhension du concept de destination, notamment urbaine, est proposée, basée sur la notion de projet et montrant la destination comme un ensemble de " projets " différenciés, mais intégrés à un " projet " global. 


\section{Le concept de destination Diversité sémantique et réalité organisationnelle}

\author{
Boualem KADRI, Ph.D. \\ Professeur \\ Département d'études urbaines et touristiques \\ Université du Québec à Montréal (ESG-UQAM) \\ kadri.boualem@uqam.ca \\ Mohamed Reda KHOMSI \\ Enseignant-doctorant en études urbaines \\ Département d'études urbaines et touristiques \\ Université du Québec à Montréal (ESG-UQAM) \\ khomsi.mohamed_reda@uqam.ca
}

\author{
Maria BONDARENKO \\ Doctorante en sémiologie \\ Université du Québec à Montréal (UQAM) \\ maria_bondarenko@hotmail.com
}

RÉSUMÉ: Le concept de destination fait l'objet d'une grande utilisation en tourisme, tant dans le monde professionnel que dans celui de la communauté scientifique. Cette situation montre toutefois un paradoxe : si l'on reconnaît la puissance du mot destination relative notamment à sa richesse anthropologique, l'on observera néanmoins un déficit définitionnel, voire l'absence d'une définition partagée par la communauté scientifique. À quelles significations renvoie le mot destination? Le présent article expose les résultats d'une analyse sémantique et conceptuelle du concept de destination réalisée à partir d'un corpus d'écrits portant plus particulièrement sur le tourisme urbain. À la lumière de cette analyse, une nouvelle perspective d'appréhension du concept de destination, notamment urbaine, est proposée, basée sur la notion de projet et montrant la destination comme un ensemble de «projets» différenciés, mais intégrés à un «projet» global.

Mots-clés : Destination, destination urbaine, analyse sémantique, projet.

Le succès du tourisme est étroitement associé au mot destination, qui évoque tout à la fois un lieu à voir, l'objet d'un désir ou d'un rêve, et une organisation capable de réaliser ce rêve. Ce terme fait aussi l'objet d'une importante utilisation tant par les organismes, par les entreprises touristiques et par les acteurs du développement que par les chercheurs en tourisme qui ne partagent pas une définition commune et reconnue du concept de destination. Ce concept devient l'objet d'un enjeu scientifique entre les disciplines exerçant une domination sur le champ du tourisme. Quelle est la signification du mot destination utilisé dans les écrits scientifiques sur le tourisme? Cette recherche vise la réalisation d'une analyse sémantique et d'une analyse conceptuelle du concept de destination à partir d'écrits portant sur le tourisme urbain.

Prolongeant la réflexion de l'Équipe MIT (Mobilité, itinéraires, tourisme, 2002, 2005) et celle de Violier (2009) invitant à considérer la destination dans une perspective de "projet», nous admettons que la destination notamment urbaine est une succession de "projets» différenciés, mais organisée et intégrée en un "projet» plus global. Peut-on retrouver ce processus à travers les écrits sur le tourisme, et plus particulièrement ceux concernant le tourisme urbain?
Comment les approches théoriques sur la destination peuvent-elles nous éclairer sur la signification du concept luimême? Deux démarches ont été privilégiées. D'une part, nous avons fait une analyse de type conceptuelle basée sur les définitions de dictionnaires et les approches théoriques liées à la destination provenant d'auteurs francophones (actes du colloque des Rendez-vous Champlain 2008, et revues Téoros et Espaces) et anglophones (revue Annals of Tourism Research). 25 documents ont été utilisés pour cette première analyse.

D'autre part, nous avons entrepris une analyse sémantique du concept de destination à partir d'un corpus constitué de 73 documents francophones portant sur le rapport entre le tourisme et l'urbain. La méthode générale de l'analyse sémantique se rapporte aux principes de la linguistique distributionnelle fondée par Bloomfield (1933; voir également Ducrot et Schaeffer, 1999:60-67) et développée dans le cadre de plusieurs courants linguistiques, dont celui de la linguistique appliquée. La sémantique distributionnelle assure une base linguistique à l'analyse sémantique automatique (assistée par ordinateur) des données textuelles. Appliquée au domaine du lexique, la sémantique distributionnelle postule que les termes ayant une distribution (entourage) contextuelle semblable possèdent une ressemblance au niveau de la 
signification. Ainsi, à travers l'étude des cooccurrences d'un terme et des contextes favorisant (ou non) son apparition, il devient possible de saisir son comportement sémantique et de préciser sa signification. Lorsque l'on fait une étude de textes scientifiques, cette méthode permet de s'abstraire des opinions particulières des experts afin de se concentrer sur le discours - du point de vue sémantico-linguistique - que ces derniers utilisent pour construire leurs énoncés. Cette méthode permet également d'explorer la sémantique de la terminologie scientifique dans le cas où le corpus mis en traitement ne comporte pas de définitions explicites des termes qu'il renferme. L'étude du concept destination a été effectuée à l'aide de Sémato, logiciel en ligne d'assistance sémantique à la catégorisation et à l'exploration des données textuelles, développé à l'Université du Québec à Montréal (voir la description détaillée sur http://semato.uqam.ca). Les résultats de cette analyse sont présentés dans la partie 2 et partiellement dans la partie 3 .

\section{La destination : complexité conceptuelle et réalités organisationnelles Une richesse anthropologique}

Avant d'être traduit en projets économique, d'aménagement, organisationnel et urbain, le mot destination renvoie à un important substrat anthropologique. Le dictionnaire étymologique en ligne (CNRTL, 2009) nous fait observer que son origine (XII siècle) est liée à l'idée de "prédestination ", une fin déjà programmée à l'avance. Au XVII ${ }^{e}$ siècle, cette signification sera accolée à un objet et à sa fonctionnalité : «Emploi auquel une chose est destinée.» Au XVIII ${ }^{e}$ siècle, elle est plutôt considérée comme un «lieu où l'on doit se rendre», coïncidant ainsi avec la naissance du tourisme en Europe». Ce terme nous renvoie déjà à une représentation de totalité exprimée par l'existence de plusieurs significations, mais surtout par la présence de deux dimensions, celles d'origine et de finalité. Cette représentation donne toute la puissance au concept, au point d'imposer un certain déterminisme. En effet, le philosophe Onfray (2007) nous fait observer que le désir de voyage prend ses origines à la fois dans l'expérience primitive du ventre maternel et dans le récit mythologique construit par les figures de Caïn et Abel en tant qu'archétypes du berger (voyageur) et du paysan (sédentaire). Ainsi, « [r] êver une destination, c'est obéir à l'injonction qui en nous parle une voix étrangère. Car un genre de démon socratique formule et trace à notre place cet éclair qui calcine en notre for intérieur l'indécis, l'imprécis et le confus» (Onfray, 2007 : 22). La destination devient l'alpha et l'oméga dans le tourisme, mais aussi l'objet ou même le projet par lequel une vie mériterait de s'achever "en beauté» comme le montre les deux personnages principaux du film The Bucket List, de Rob Reiner (2007). Ces derniers, se sachant condamnés par la maladie, dressent alors une liste de choses à réaliser avant la fin et parmi lesquelles l'on retrouve le désir de visiter des destinations mythiques (les pyramides d'Égypte, la muraille de Chine) et des villes (Hong Kong, Saint-Tropez).

Cette richesse anthropologique ne se reflète guère dans les dictionnaires spécialisés, exprimant alors une insuffisance définitionnelle.

\section{Une insuffisance définitionnelle}

Si certains dictionnaires de la langue française reprennent les diverses significations du mot destination (destinée, usage, direction, lieu), les dictionnaires spécialisés en tourisme et en géographie sont pour certains d'entre eux muets sur la définition ou en donnent une explication très réduite. Ainsi, le Dicotour (Olivier, 2006) n'accorde pas de place au mot destination tandis que les dictionnaires de géographie limitent sa définition à l'idée d'une place choisie à l'avance : le Dicotour en ligne du site de GeotourWeb met l'accent sur le «lieu où se rendre». Les Mots de la géographie (Brunet, 1993 : 154) considèrent la destination comme «un lieu vers lequel on se dirige pour s'y arrêter », tout en rejetant les significations implicites de «but» et de "vocation» contenues dans le mot lui-même, car ne relevant pas de la géographie.

La définition la plus élaborée du mot destination est fournie par l'Encyclopedia of Tourism (Jafari, $2000: 144-145$ ) :

Destination, as distinct from origin or market, refers to the place where tourist intend to spend their time away from home. This geographical unit visited by tourists may be a self-contained centre, a village or a town or a city, a region or an island or a country. Furthermore, a destination may be a single location, a set of multi-destinations as part of a tour, or even a moving destination such as a cruise.

Considérant la destination comme un concept large, diversifié et complexe à définir, la définition met toutefois l'accent sur l'aspect géographique (lieu) et sur celui du fonctionnement en termes économique (marché, produit) et psychosociologique (motivation du consommateur).

Si l'on ne retrouve pas une définition complète et commune à tous les chercheurs, ces derniers n'en élaborent pas moins des approches théoriques liées à l'objet destination. Ces approches peuvent-elles nous éclairer davantage sur la destination et sa signification?

\section{Un réductionnisme explicatif, mais aussi une perspective systémique}

Le concept de destination pris dans sa dimension de totalité et de complexité pose-t-il un problème au point où sa définition se voit constamment ajustée par un certain réductionnisme? Cette section se penchera sur les approches de la destination développées par des auteurs francophones et anglophones.

Deux constats peuvent être déduits de la littérature choisie et traitant du concept de destination. D'une part, ce dernier semble devenu un mot-valise mythique (Hazebroucq, 2009 : 8) dont la signification est fortement corrélée à des considérations contextuelles. D'autre part, la domination scientifique de deux disciplines, en l'occurrence la géographie et le marketing, impose sa façon d'aborder ce concept. Malgré la nature de totalité et de complexité du phénomène touristique, le concept de destination reste attaché à une dimension spatiale, car les pratiques touristiques se définissent aussi par la mobilité et le déplacement (Violier, 2009). Si l'approche par la géographie est la plus dominante au sein de la littérature francophone, c'est parce que les partisans de cette discipline considèrent la destination d'abord comme un lieu. Les définitions, telles que celle de l'Équipe MIT (2005: 11), montrent clairement le poids de la variable spatiale dans l'interprétation de ce concept : «La 
Tableau 1 : Évolution des attributs étudiés dans la définition du concept de destination

\begin{tabular}{|c|c|c|c|c|c|c|c|c|c|c|c|c|c|c|}
\hline \multirow{3}{*}{$\begin{array}{l}\text { Documents mis } \\
\text { en analyse } \\
\text { conceptuelle }\end{array}$} & \multicolumn{14}{|c|}{ Attributs étudiés dans la définition du concept de la destination } \\
\hline & 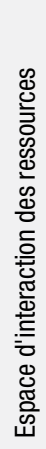 & 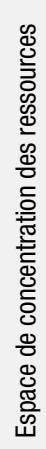 & 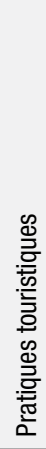 & 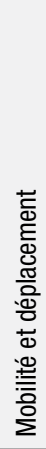 & 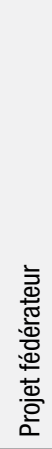 & 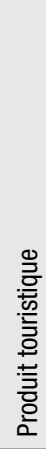 & 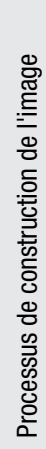 & 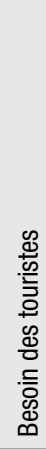 & 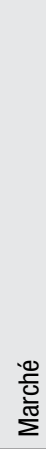 & 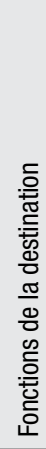 & 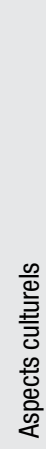 & 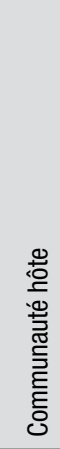 & 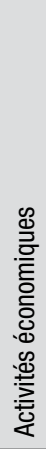 & 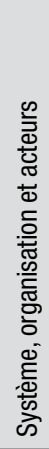 \\
\hline & \multicolumn{5}{|c|}{ Communauté hôte } & \multicolumn{6}{|c|}{ Activités économiques } & \multicolumn{3}{|c|}{ Approche systémique } \\
\hline Flament (1975) & & $\mathrm{x}$ & & & & & & $\mathrm{x}$ & & & & & & \\
\hline Weber (1979) & & $\mathrm{x}$ & & & & & & & & & & & & \\
\hline Butler (1980) & & & & & & $x$ & & & & & & & & \\
\hline Stabler (1988) & & & & & & & $\mathrm{x}$ & & & & & & & \\
\hline Telisman-Kosuta (1989) & & & & & & & $\mathrm{x}$ & & & & & & & \\
\hline Kye-Sung (1990) & & & & & & & $\mathrm{x}$ & & & & & & & \\
\hline Leiper (1990) & & & & & & & $x$ & & & & & & & $x$ \\
\hline Etchner et Ritchie (1991) & & & & & & & $\mathrm{x}$ & & & & & & & \\
\hline Gordon et Goodall (1992) & & & & & & & & & & & & & $x$ & $x$ \\
\hline Gun (1994) & & $\mathrm{x}$ & & & & & & $x$ & & & & & & \\
\hline Tocquer et Zins (1999) & & & & & & $x$ & & & & & & & & $x$ \\
\hline Davidson et Maitland (2002) & & $x$ & & & & $x$ & $x$ & $x$ & & $x$ & $x$ & & & \\
\hline Gallarza et al. (2002) & & & & & & & $x$ & & & $x$ & & & & \\
\hline JafarJafari (2000) & & $x$ & & & & & & $x$ & $x$ & & & & & $x$ \\
\hline Knafou et Stock (2003) & & & $x$ & & $x$ & & & & & & & & & $x$ \\
\hline Équipe MIT (2005) & $x$ & & $x$ & & & & $x$ & $x$ & & & $x$ & & & \\
\hline Weaver et al. (2007) & & & & & & & & $x$ & & & & & & \\
\hline Ceriani et al. (2008) & & & $x$ & & $x$ & & & & & & & & & $x$ \\
\hline Goeldner et Ritchie (2006) & & $x$ & & & & $x$ & $x$ & & & $x$ & & $x$ & $x$ & $x$ \\
\hline Botti et al. (2008) & & & & & & & & & & & & & $x$ & $x$ \\
\hline Violier (2009) & & & $x$ & $x$ & & & $x$ & & & & & & & \\
\hline Hazebroucq (2009) & & $x$ & & & $x$ & $x$ & & & & & & & & $x$ \\
\hline Lesne et Zins (2009) & & $x$ & & & & & & & & & & & & $x$ \\
\hline
\end{tabular}

Source : compilation des auteurs. 
destination est un lieu d'interactions territoriales qui relient ensemble les données socioculturelles, naturelles, patrimoniales et techniques».

Selon Hayllar et al. (2008), l'étude des territoires touristiques concerne d'abord l'étude des espaces. L'importance du caractère géographique dans l'appréhension du concept de destination pose toutefois certains problèmes, dont ceux rapportés par Violier (2009 : 27), par exemple :

Les approches de tendance positiviste qui tentent de définir les lieux touristiques par la présence d'objets naturels ou culturels aboutissent à la construction d'inventaires vite pris en défaut, car la présence d'objets ne suffit pas à assurer la présence des touristes [...] La destination serait ce que les touristes perçoivent comme l'espace où ils mettront en œuvre des pratiques qui définissent alors le territoire touristique.

Dans le même sens, Ceriani et al. (2008) «considèrent qu'un lieu est touristique par les touristes qui le pratiquent et que, dialogiquement, il correspond plus ou moins au projet formé par les touristes». En d'autres mots, la destination serait la mise en acte d'un projet fédérateur des pratiques touristiques mises en œuvre par tous les intervenants au sein de la destination.

Si certains soubassements disciplinaires restent fondés sur la géographie, nous avons noté toutefois la prise de conscience d'une nécessité d'intégrer de nouveaux éléments gravitant désormais autour de l'espace, du territoire ou du lieu touristique. Dans cet esprit, l'Équipe MIT (2005: 8) a tenté de croiser le temps et l'espace en définissant le «moment de lieu qui agit comme un embrayage à un moment donné et à un endroit donné entre acteurs, pratiques goûts esthétiques, valeurs sociales, normes juridiques, économiques et techniques».

Au-delà de l'espace, la destination peut être perçue comme un produit et un système. Si cette perspective initiée par le marketing ne rejette pas la dimension spatiale du terme, elle en adopte toutefois un angle de lecture différent de la géographie, notamment à travers le modèle de cycle de vie des espaces touristiques développé par le géographe Richard Butler (1980). Ce dernier présentait déjà en 1980 ce modèle, inspiré de la théorie du cycle de vie du produit, mettant en perspective la naissance et le déclin de la destination en fonction de l'évolution du nombre des visiteurs dans le temps. Longtemps critiqué, essentiellement à cause de son caractère générique, ce modèle reste néanmoins le plus utilisé pour évaluer les performances d'une destination touristique. Dans cette même logique, à savoir la destination en tant que produit, Tocquer et Zins (1999) définissent la destination touristique comme un type de produits et services touristiques issus de nombreux opérateurs privés et publics. Davidson et Maitland (2002) vont dans le même sens et considèrent la destination comme un lieu de production de produits touristiques concentrant l'attention et les motivations des visiteurs.

D'autres auteurs comme Kye-Sung (1990), Etchner et Ritchie (1991), Stabler (1988), Telisman-Kosuta (1989), Gallarza et al. (2002) définissent la destination à partir de l'image que cette dernière renvoie à ses visiteurs. Dans cette perspective, la destination peut être un site, un village, une ville, une région, un pays ou une zone. La territorialité de la destination dépendra à la fois de l'effort de communication entrepris par cette dernière en sus des expériences vécues. De ce point de vue, le concept acquiert une dimension vitale dans la construction identitaire du territoire, dans la mesure où «la destination devient un lieu qui prend la dimension de destination », la marque de ce lieu (Gibson et Tiard, 1999 : 79).

En complément des deux logiques présentées ci-dessus, nous observons une approche systémique perçue comme une perspective à la fois holistique et pluridisciplinaire. Dans cet esprit, Knafou et Stock (2003) perçoivent le lieu touristique comme indissociable de l'intention du touriste : le déplacement caractéristique de la pratique touristique est saisi comme la mise en acte d'un "projet de recréation ». À cet effet, la destination est considérée par Botti et al. (2008: 20) «comme un système dans lequel interagissent de nombreuses organisations qui appartiennent à la même branche d'activité ou à des branches d'activités différentes». Cette référence à la diversité d'intervenants est reprise par Goeldner et Ritchie (2006) dans leur tentative d'identification des différentes composantes de la destination touristique. Toutefois, la forme circulaire du modèle proposée par ces deux auteurs renvoie à son tour à la difficulté de délimiter une destination.

La connaissance du concept de destination a évolué depuis les années 1980, notamment en termes de perspective. En se basant sur un corpus pluridisciplinaire de 25 textes, trois perspectives sont identifiées : géographique, marketing et systémique. Le tableau 1 nous montre que les dimensions fonctionnelles du concept ont été enrichies à partir des années 1990, où la tendance a été celle de l'adoption des approches croisant plusieurs disciplines afin de cerner un concept accentuant son flou et sa complexité avec l'évolution de l'activité touristique. La littérature examinée permet de relever une volonté explicite d'enrichir la définition de la destination et son appréhension, notamment selon une approche holistique. Cette dernière considère la destination comme un projet indissociable et non fragmentaire, et a un caractère conciliateur des approches disciplinaires et de dépassement d'une analyse souvent réduite à la perspective géographique ou mercatique. Ce résultat, qui contribue à affranchir le terme du flou fonctionnel et sémantique, mérite d'être complété par une analyse sémantique du concept de destination tel qu'utilisé dans les écrits francophones relatifs au tourisme urbain.

\section{L'analyse sémantique du concept de destination La destination comme objet de l'étude sémantique : méthodes et hypothèse}

L'analyse sémantique du concept de destination implique plusieurs approches et étapes.

D'une part, au centre de l'investigation assistée par ordinateur se trouve le mot lemmatisé destination, dont 347 occurrences (occ.) sont relevées sur les 73 documents analysés. Une attention particulière a été portée à certaines "synapsies", expressions à la base du terme destination pertinentes pour notre analyse (voir tableau 2).

D'une autre part, et afin d'établir les limites sémantiques du terme destination, nous avons mis en analyse une trentaine de concepts complémentaires, que le logiciel avait suggérés comme pertinents, du point de vue de leur compatibilité contextuelle (occurrence) avec le mot destination. 
Tableau 2: Synapsies du terme destination devenus l'objet de l'analyse sémantique

\begin{tabular}{l|c|c}
\hline $\begin{array}{l}\text { Synapsies du terme } \\
\text { destination pertinentes pour l'analyse }\end{array}$ & Nombre d'occurrences & $\begin{array}{c}\text { Pourcentage : } \mathbf{3 4 7} \text { (la totalité de l'apparition du terme } \\
\text { destination dans le corpus) }=\mathbf{1 0 0} \%\end{array}$ \\
\hline Destination touristique & 71 & 20,4 \\
\hline Destination touristique urbaine & 15 & 12,6 \\
\hline Destination urbaine & 44 & 2,3 du total de 71 occ. de destination touristique] \\
\hline Destination métropolitaine & 8 & 1,4 \\
\hline Destination-ville & 5 & 1,1 \\
\hline Destination de/du tourisme & 4 & 1,1 \\
\hline Ville de destination & 4 & 1,1 \\
\hline Destination culturelle & 4 & 0,6 \\
\hline Ville-destination & 2 & 0,6 \\
\hline Destination [de] jeu & 2 & 0,3 \\
\hline Destination congressuelle & 1 & 0,3 \\
\hline Destination d'accueil de réunions internationales & 1 & \\
\hline Destination affaires & 1 & \\
\hline Source : compilation des auteurs à partir des données générées par le logiciel Sémato. & \\
\hline
\end{tabular}

Enfin, l'analyse des données sur les cooccurrences a été guidée par certaines prémisses tirées de la sémantique du terme destination développée dans la langue française. Le français contemporain distingue deux groupes de significations du mot destination: Le premier comporte une série de sens remontant à l'origine du mot latin destinatio, venu dans la langue française à la fin du XII ${ }^{\mathrm{e}}$ siècle avec la signification « fin, finalité ». Ces anciennes significations, à cause de leur caractère métaphysique, ne paraissent pas pertinentes, à première vue, pour le discours scientifique sur le tourisme. Par contre, le second groupe, beaucoup plus récent, est en lien immédiat avec ce dernier : il s'agit de "l'emploi auquel une chose est destinée» (fixée en 1690), et du «lieu où l'on doit se rendre» (en 1770) (Rey, 1992 : 589). Ainsi, la structure sémantique du mot destination s'organise autour de deux pôles :

\section{LIEU OÙ SE RENDRE}

Comment ces pôles se manifestent-ils dans le discours sur le tourisme? L'exploration sémantique nous amène à l'hypothèse suivante :

A) La signification du terme destination dans le discours sur le tourisme se caractérise par une fusion des pôles sémantiques lieu et emploi. Il n'est pas possible de les dissocier sans briser le sens et la valeur terminologique du mot.

B) On constate un déficit et en même temps une portée définitionnelle du terme destination. La fusion en question ne peut être reconnue comme incontestablement délibérée. Parmi tous les énoncés comportant le mot destination, il n'y en a aucun qui constitue une définition explicite du terme en question. Il n'empêche que le terme destination, et plus spécifiquement destination touristique (urbaine), demeure très important pour la définition des termes fondamentaux en tourisme. Le mot destination se retrouve souvent dans des énoncés de type définitionnel, où il joue le rôle d'attribut auprès du sujet ville/métropole selon le modèle suivant :

Ville/métropole comme.../en tant que.../constitue.../ est une.../émerge /s’impose comme.../devient/aspirer de devenir.../etc. destination touristique

C) Malgré l'impossibilité de dissocier les composantes lieu et emploi, le rapport entre elles n'est pas une constante. Chaque occurrence du mot destination révèle une gravité vers l'un de ces pôles. Cette gravité n'est pas négligeable du point de vue conceptuel, méthodologique et épistémologique. Cela donne lieu à distinguer plusieurs variétés sémantiques du terme destination.

\section{À la recherche des moyens de saisir} des variétés sémantiques de la destination

Pour récupérer les variétés sémantiques du terme destination, avec l'absence de définitions explicites, nous avons réuni deux procédures : l'étude sur l'entourage sémantique et la grille des repérages divers.

Concernant l'entourage sémantique, chaque variété du terme destination s'associe à un ensemble des termes cooccurrents dans un contexte défini (phrase, paragraphe, document, etc.). Le tableau 3 permet de comparer les cooccurrences du terme destination avec d'autres groupes thématiques dans le contexte de la phrase. Plus grand est l'écart entre le nombre de cooccurrences récupérées dans le corpus et celui reconnu comme "attendu», plus fort est le lien sémantique entre deux concepts, et par conséquent plus forte est leur capacité de se définir. On distingue des contextes favorables à l'utilisation du terme destination et des contextes peu ou pas favorables, tels qu'illustrés ici (selon un ordre décroissant) : 
Tableau 3: Cooccurrences phrastiques du terme destination avec des thèmes du corpus

\begin{tabular}{|c|c|c|c|c|}
\hline \multirow[b]{2}{*}{$\begin{array}{l}\text { THÈMES } \\
\text { (ensembles de termes réunis } \\
\text { selon leur parenté sémantique) }\end{array}$} & \multirow[b]{2}{*}{ 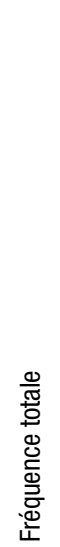 } & \multicolumn{3}{|c|}{$\begin{array}{l}\text { COOCCURRENCES } \\
\text { par phrase avec le terme } \\
\text { DESTINATION } \\
\text { (347 occurrences totales) }\end{array}$} \\
\hline & & 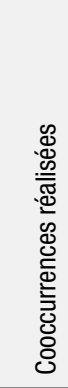 & 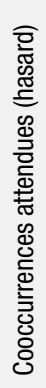 & 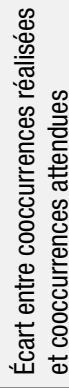 \\
\hline SE RENDRE/SE DÉPLACER/DÉPLACEMENT & 77 & 6 & 2 & 4 \\
\hline VOYAGE & 175 & 7 & 3 & 4 \\
\hline USAGE/UTILISATION & 30 & 2 & 1 & 1 \\
\hline APPROPRIATION & 34 & $\emptyset$ & $\emptyset$ & $\emptyset$ \\
\hline FONCTION TOURISTIQUE & 39 & 0 & $\emptyset$ & $\emptyset$ \\
\hline FONCTION URBAINE & 22 & 0 & $\emptyset$ & $\emptyset$ \\
\hline HABITER/VIVRE TOURISTIQUEMENT & 46 & 0 & $\emptyset$ & $\emptyset$ \\
\hline TOURISTICITÉ & 41 & $\emptyset$ & $\emptyset$ & $\emptyset$ \\
\hline URBANITÉ & 182 & $\emptyset$ & $\emptyset$ & $\emptyset$ \\
\hline PRATIQUE TOURISTIQUE & 135 & 5 & 3 & 2 \\
\hline ACTIVITÉ TOURISTIQUE & 93 & 4 & 2 & 2 \\
\hline MOBILITÉ & 161 & 5 & 3 & 2 \\
\hline OFFRE & 255 & 17 & 5 & 12 \\
\hline PRODUIT & 227 & 14 & 5 & 9 \\
\hline AMÉNAGMENT/AMÉNAGER & 200 & 4 & 4 & 0 \\
\hline ÉQUIPEMENT/ÉQUIPER & 201 & 8 & 4 & 4 \\
\hline GESTION/GÉRER & 198 & 22 & 5 & 17 \\
\hline PLANIFICATION/PLANIFIER & 215 & 8 & 4 & 4 \\
\hline ORGANISATION/ORGANISER & 127 & 12 & 6 & 6 \\
\hline PROJET & 320 & 7 & 6 & 1 \\
\hline POLITIQUE & 443 & 13 & 9 & 4 \\
\hline IMAGE & 289 & 24 & 6 & 18 \\
\hline IDENTITÉ & 82 & 1 & $\emptyset$ & $\emptyset$ \\
\hline QUALITÉ & 262 & 30 & 5 & 25 \\
\hline MOTIVATION & 270 & 7 & 6 & 1 \\
\hline ATTRACTIVITÉ & 466 & 23 & 9 & 14 \\
\hline DIVERSITÉ & 413 & 19 & 8 & 11 \\
\hline CONCURRENCE/COMPÉTITION & 155 & 11 & 3 & 8 \\
\hline CLASSEMENT & 58 & 5 & 1 & 4 \\
\hline DÉVELOPPEMENT & 347 & 24 & 10 & 14 \\
\hline DEVENIR & 330 & 19 & 7 & 12 \\
\hline
\end{tabular}

Source : compilation des auteurs à partir des données générées par le logiciel Sémato.
L'entourage favorisant :

- qualité, gestion, image;

- attractivité, développement, offre, produit, devenir, diversité, concurrence;

- équipement, planification, politique, organisation,

classement;

- se déplacer, se rendre/déplacement, voyage;

- projet, motivation, aménagement, pratique touristique, activité touristique, mobilité.

L'entourage peu ou pas favorable :

- usage/utilisation du lieu, appropriation (du lieu);

- habiter/vivre touristiquement (le lieu);

- fonction touristique, fonction urbaine;

- touristicité, urbanité.

De plus, il est intéressant de voir comment les termes mentionnés comme favorables sont liés entre eux. Cela nous permet de déterminer la place du concept de destination dans les réseaux de rapports mutuels. L'illustration 1 offre une présentation visuelle des rencontres mutuelles de ces termes (contexte de la phrase).

L'on observe que le terme destination fait partie de plusieurs réseaux sémantiques. Cela donne lieu à parler de plusieurs branches sémantiques à l'intérieur du concept destination : chaque réseau peut être associé à une branche d'une manière présentée dans le tableau 4.

Or, les réseaux détectés ne sont pas suffisamment nets pour formuler les variétés sémantiques de destination de manière claire. Pour les ajuster et systématiser davantage, nous avons créé une grille des repérages sémantiques. Cette grille est basée sur la distinction primaire «lieu où se rendre» vs «emploi du lieu ", et constitue des applications aux domaines sémantiques suivants :

- Repérage spatial : destination d'un déplacement vs localisation.

- Focus général : vision de l'extérieur vs vision de l'intérieur.

- Focus spécifique (point de vue d'acteur) : Acteur voyageant (consommateur, choisissant, acceptant de se faire attirer et envoyer, habitant, appropriant, habitant touristiquement le lieu) vs Acteur intermédiaire (envoyant, organisant, offrant, imposant, attirant, vendant, promouvant l'image) vs Acteur accueillant (récepteur, offrant, imposant, attirant, aménageant, développant, effectuant une politique et des projets).

- Repérage pragmatique (type de motivation) : motivation du déplacement vs motivation (fonction) de l'usage/aménagement du lieu.

- Repère taxinomique/axiologique: rapport avec un classement.

- Repérage métascientifique : rapport avec une définition des concepts fondamentaux de la science touristique.

- Repérage épistémologique : phénomène vu comme naturel vs phénomène vu comme construit.

La combinaison de deux approches permet de distinguer les variétés du concept de destination suivantes : destination I = direction du déplacement; destination II = direction de l'envoi (organisé); destination III-A = lieu (partie de la ville) touristiquement aménagé; destination III-B = lieu (partie de la ville) touristiquement utilisé, partagé et habité; destination IV = projet du lieu (ville entière) touristique; destination $\mathrm{V}=$ destinée. 


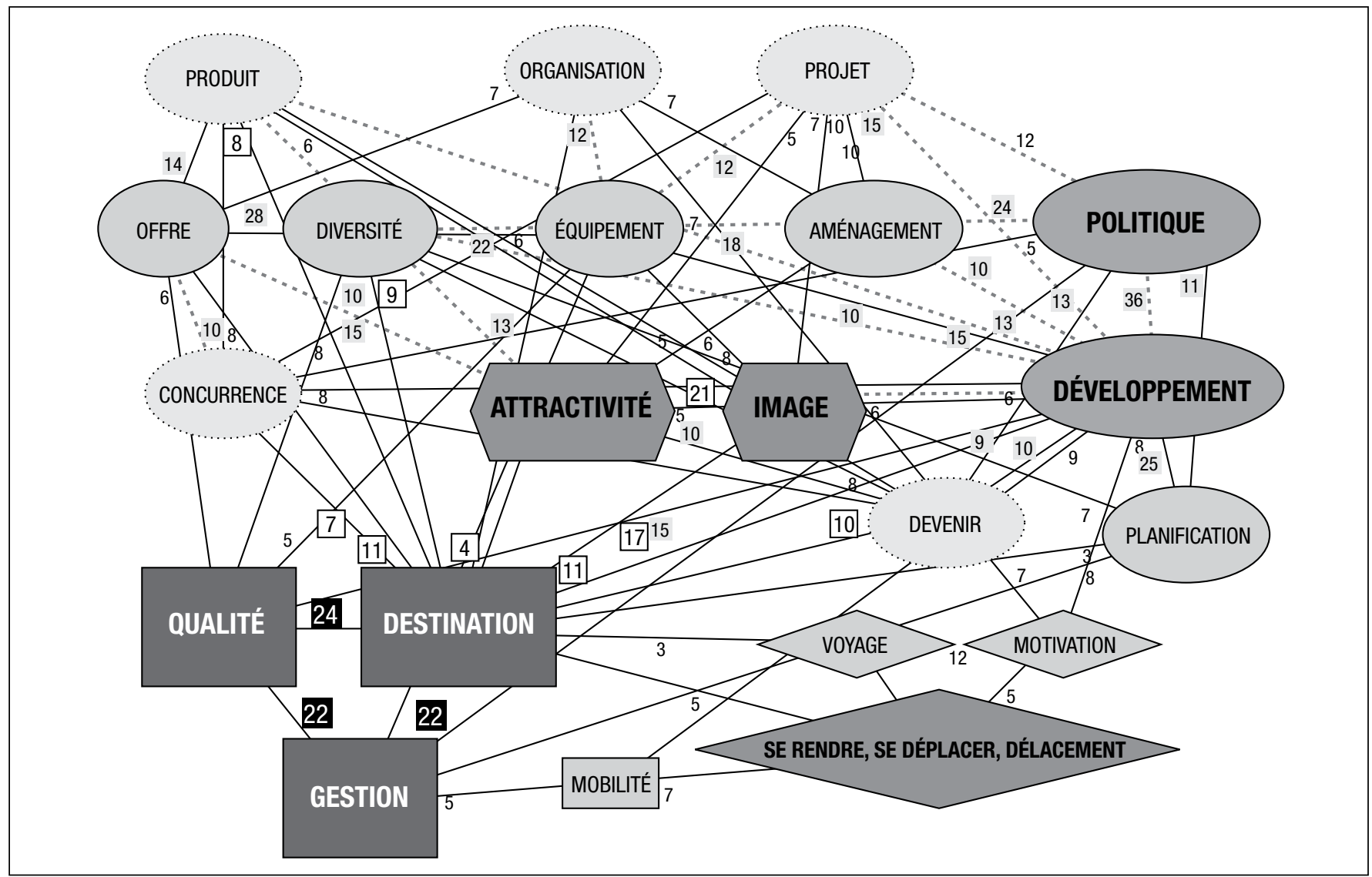

ILLUSTRATION 1 : Graphique des réseaux de cooccurrences des thèmes du corpus (source : généré par le logiciel Sémato à partir du corpus étudié).

\section{Description des variétés sémantiques du concept de destination}

Le tableau 5 présente les variétés distinguées et leur caractéristique selon le rapport aux réseaux sémantiques ajustés par la grille des repérages.

\section{Destination comme direction du déplacement (variété I)}

L'acception I se caractérise par l'entourage sémantique se rendre/se déplacer/déplacement/ voyage.

La particularité de cette acception est sa tendance vers le pôle «lieu où se rendre». L'optique qui lui est propre correspond à celle de l'acteur voyageant (touriste). L'idée du déplacement physique mise en valeur ici s'actualise par la cooccurrence avec les termes signifiant les moyens de transport. Liée à l'idée de "déplacement physique des personnes ", l'acception I présente la destination comme un phénomène «naturel». Elle donne l'accès à deux classifications des destinations parmi huit variétés que nous avons relevées dans notre corpus : (A) par le caractère géographique du lieu (destination touristique urbaine vs destinations touristiques non urbaines); (B1) par la motivation du touriste (destination d'affaires, de jeu, d'accueil de congrès, etc.). L'acception I permet de définir des concepts fondamentaux du discours scientifique touristique (tels ceux de tourisme et touriste) selon le modèle suivant : idée de déplacement + idée de motivation (motif) du déplacement. Nous avons attesté au minimum de 17 définitions explicites aussi bien qu'implicites qui suivent ce modèle :

Personne se déplaçant temporairement vers des lieux situés dans l'espace-temps du hors-quotidien afin d'y développer des pratiques récréatives (010-254-1) ${ }^{1}$.

En ouvrant une fenêtre spatio-temporelle inédite (le déplacement temporaire à des fins de récréation), le tourisme a créé les conditions pour que les individus s'affranchissent de certains cadres communautaires, en privilégiant le «souci de soi» (036-2038-4).

Pourtant l'acception I n'est pas dominante. Le nombre d'utilisations du terme destination dans le sens direction de déplacement dépasse à peine six : «se déplacer/venir vers la destination» (3 occ.), «aller à la destination» (1 occ.), «se rendre dans une destination» (2 occ.). Pour parler de la direction du déplacement dans le sens purement physique, les auteurs préfèrent utiliser d'autres outils lexicaux. Si le terme destination n'est pas beaucoup utilisé dans le contexte de déplacement physique et par conséquent dans les définitions du tourisme basées sur l'opposition des composants déplacement et motivation, n'est-ce pas parce qu'il est voué à saisir la fusion inséparable des composants en question?

Le reste des termes mentionnés comme contextes favorables au terme destination forme un ensemble qui couvre les 
Tableau 4: Réseaux sémantiques dans leur rapport à des significations potentielles du terme destination

\begin{tabular}{|c|c|}
\hline Description des réseaux & $\begin{array}{l}\text { Branches sémantiques potentiellement associées } \\
\text { aux réseaux }\end{array}$ \\
\hline $\begin{array}{l}\text { a. Le plus distinguable est une chaîne fermée de trois termes fermement liés entre eux (cf. rectangles foncés } \\
\text { sur le SCHÉMA 1) : DESTINATION-(22)-GESTION-(22)-QUALITÉ-(24)-[DESTINATION] } \\
\text { (Le chiffre est l'indicateur du nombre des cooccurrences phrastiques entre deux termes voisins.) }\end{array}$ & $\begin{array}{l}\text { Destination comme produit de gestion visant } \\
\text { l'excellence et la qualité }\end{array}$ \\
\hline b. Un autre réseau : DESTINATION-(17)-IMAGE-(22)-ATTRACTIVITÉ-(13)-PRODUIT-(8)-[DESTINATION]. & Destination comme image et attractivité \\
\hline 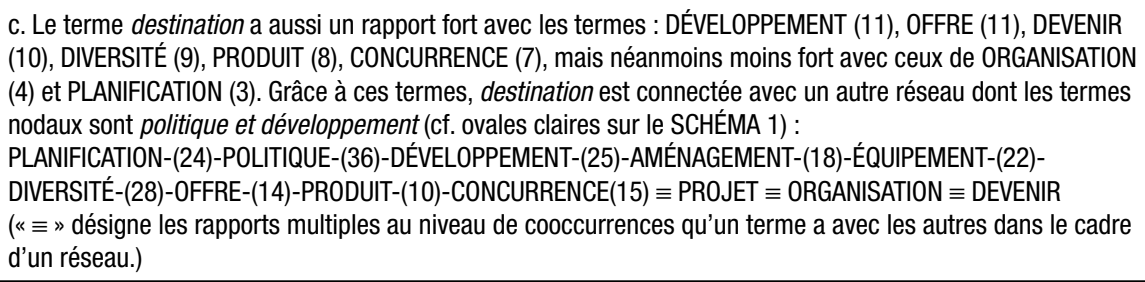 & $\begin{array}{l}\text { Destination comme projet de développement et } \\
\text { politique de planification (incluant l'équipement } \\
\text { et aménagement) dans le but de créer — dans la } \\
\text { condition de concurrence - une offre et un produit } \\
\text { (touristiques) diversifiés et compétitifs }\end{array}$ \\
\hline $\begin{array}{l}\text { d. La destination fait également partie du réseau DESTINATION-(3)-VOYAGE-(12)-MOTIVATION-(5)-SE } \\
\text { RENDRE-(5)-[DESTINATION] (cf. losanges sur le SCHÉMA 1) }\end{array}$ & $\begin{array}{l}\text { Destination comme déplacement dans un certain } \\
\text { but (motivation) }\end{array}$ \\
\hline $\begin{array}{l}\text { e. L'extension possible de la même chaîne (d.) donne DESTINATIOIN-(3)-VOYAGE-(12)-MOTIVATION-(5)-SE } \\
\text { RENDRE-(7)-MOBILITÉ-(5)-GESTION-(22)-[DESTINATION]. }\end{array}$ & $\begin{array}{l}\text { Destination comme organisation (gestion) du } \\
\text { déplacement dans un certain but (motivation) }\end{array}$ \\
\hline
\end{tabular}

acceptions II, III-A, IV et V. Pourtant, le noyau de cet ensemble est situé dans l'espace destiné aux acceptions II, III-A (la destination comme lieu [partie de la ville] aménagé) et IV (destination comme lieu [ville entière] résultant d'une politique qui domine dans le discours sur le tourisme).

\section{Destination comme lieu touristiquement aménagé (variété III-A) et touristiquement utilisé/habité/partagé (variété III-B), et destination comme projet de la ville destinée au tourisme (variété IV)}

La particularité que les acceptions III-A, III-B et IV ont en commun consiste en leur tendance vers le pôle de l'«emploi du lieu ", d'où l'optique représentant le lieu d'accueil de l'intérieur, du point de vue des acteurs chargés de la gestion, de l'aménagement et de la planification. La destination est conçue ici comme un phénomène construit. Le point de divergence entre les acceptions III et IV s'exprime dans l'opposition de deux visions : intégrale vs non intégrale. L'acception IV privilégie la vision intégrale représentant le milieu urbain (ville) tout entier comme étant sujet à des projets de développement et de planification :

Il s'agit de mettre en place une organisation qui ait la charge de la gestion stratégique du développement du tourisme urbain à l'échelle de la destination urbaine (016-1032-1).

La mise en ouvre de la gestion intégrée de la qualité des destinations touristiques urbaines suppose qu'une attention particulière soit portée à l'analyse de l'existant (033-1917-3).

L'acception III (-A, -B) privilégie la vision non intégrale du milieu urbain et oppose des zones différentes de la ville en mettant l'accent sur certaines de leurs caractéristiques propres : utilisation/fonction/aménagement/équipement.
Aujourd'hui, le quartier est devenu la nouvelle destination au cœur de Québec - restaurants aux dernières tendances, lounge dernier cri, belles boutiques avantgardistes (040-2156-6).

Si le tourisme urbain est celui qui génère le moins de circulation automobile (zone d'intérêt touristique concentrée et bonne accessibilité aux transports en commun), son développement peut entraîner d'autres nuisances (surfréquentation de certains espaces, conflits d'usage avec la population, développement de la circulation des autocars de tourisme...) surtout si la destination urbaine se place à une échelle plus large que la seule ville centre ou agglomération (016-1014-2).

À l'intérieur de l'acception III, nous distinguons deux variétés. La variété III-A est associée au contexte aménagement/équipement, il s'agit de l'aménagement touristique d'une zone saisie du point de vue de l'agent accueillant et gérant. La variété III-B est associée aux termes utiliser/approprier/habiter/vivre/partager le lieu. Elle nous envoie à l'aspect anthropologique et identitaire visant les gens qui habitent ou qui fréquentent une ville.

La particularité sémantique qui caractérise l'ensemble dominant III-A, III-B, IV se reflète directement sur la façon de classer des destinations aussi bien que de définir le tourisme. Cette façon sera sensiblement différente par rapport à celle que nous avons relevée pour l'acception I.

Ainsi, l'acception III-A permet la classification suivante (C2) : destination comme zone urbaine utilisée/aménagée touristiquement vs d'autres zones urbaines. Les acceptions III-A et IV donnent l'accès au classement des villes entières ou ses quartiers selon le type de motivation touristique (attractivité) dominante (B2) : destination d'affaires, de jeu, d'accueil de congrès, etc. L'acception IV est compatible avec les classements suivants : 


\begin{tabular}{|c|c|c|c|c|c|c|}
\hline \multicolumn{7}{|c|}{ Tableau 5: Variétés du terme destination } \\
\hline \multirow[b]{2}{*}{ CRITÈRES } & \multicolumn{6}{|c|}{ VARIÉTÉS SÉMANTIQUES (ACCEPTIONS) DU CONCEPT DESTINATION } \\
\hline & $\left.\right|_{\text {direction du }}$ & $\prod_{\substack{\text { direction de l'envoi } \\
\text { (organisé) }}}$ & \multicolumn{2}{|c|}{$\begin{array}{c}\text { III-A } \\
\text { lieu aménagé } \\
\text { III-B } \\
\text { lieu utilisé/partagé/habité }\end{array}$} & $\begin{array}{l}\text { IV } \\
\text { projet du lieu (ville entière) } \\
\text { résultant d'un projet } \\
\text { et d'une politique }\end{array}$ & $\underset{\text { destinée }}{\mathbf{V}}$ \\
\hline $\begin{array}{l}\text { PÔLE SÉMANTIQUE } \\
\text { DOMINANT }\end{array}$ & $\begin{array}{l}\text { LIEU OÙ } \\
\text { SE RENDRE } \\
\text { Pôle sémantique }\end{array}$ & $\begin{array}{l}\text { LIEU OÙ } \\
\text { L'ENVOYER }\end{array}$ & \multicolumn{2}{|c|}{$\begin{array}{l}\text { EMPLOI } \\
\text { DU LIEU }\end{array}$} & 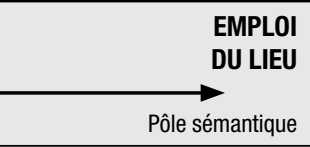 & $\begin{array}{l}\text { DESTINÉE } \\
\text { DU LIEU/DE LA } \\
\text { PERSONNE }\end{array}$ \\
\hline $\begin{array}{l}\text { FORMULATION } \\
\text { DE LA SIGNIFICATION }\end{array}$ & $\begin{array}{l}\text { Direction du déplace- } \\
\text { ment physique, lieu } \\
\text { où l'on se rend }\end{array}$ & $\begin{array}{l}\text { Direction du déplace- } \\
\text { ment (où on envoie) } \\
\text { et lieu aménagé } \\
\text { comme produit. }\end{array}$ & $\begin{array}{l}\text { Lieu (zone de la v } \\
\text { utilisé/aménagé } \\
\text { touristiquement } \\
\text { III-A }\end{array}$ & $\begin{array}{l}\text { le) destiné au tourisme } \\
\text { utilisé/habité } \\
\text { touristiquement } \\
\text { III-B }\end{array}$ & $\begin{array}{l}\text { Lieu (vision intégrale) comme } \\
\text { sujet d'un projet et d'une } \\
\text { politique du développement/ } \\
\text { aménagement touristique }\end{array}$ & $\begin{array}{l}\text { Destinée que la ville } \\
\text { doit réaliser et desti- } \\
\text { née des personnes }\end{array}$ \\
\hline $\begin{array}{l}\text { TRAIT CONSTITUANT } \\
\text { DE LA SIGNIFICATION }\end{array}$ & $\begin{array}{l}\text { L'idée de } \\
\text { déplacement physique }\end{array}$ & $\begin{array}{l}\text { L'idée d'expédition } \\
\text { et produit, et optiques } \\
\text { mixtes }\end{array}$ & \multicolumn{2}{|c|}{$\begin{array}{l}\text { Lieu (partie de la ville) vu de l'intérieur } \\
\text { à travers l'équipement/utilisation : } \\
\text { Vision non intégrale }\end{array}$} & $\begin{array}{l}\text { Lieu (ville entière) vu de } \\
\text { l'intérieur à travers le projet/ } \\
\text { développement: Vision intégrale }\end{array}$ & $\begin{array}{l}\text { Devoir } \\
\text { à accomplir }\end{array}$ \\
\hline \multirow{3}{*}{$\begin{array}{l}\text { LEXIQUE REPÉRÉ } \\
\text { DANS LE CORPUS } \\
\text { QUI «FAVORISE» } \\
\text { L'APPARITION DE LA } \\
\text { VARIÉTÉ DISTINGUÉE } \\
\text { DU TERME DESTINATION }\end{array}$} & \multirow[t]{3}{*}{$\begin{array}{l}\text { Se rendre, se déplacer, } \\
\text { aller/moyens de } \\
\text { transport, déplace- } \\
\text { ment (physique)/ } \\
\text { mouvement/voyage }\end{array}$} & \multirow[t]{3}{*}{$\begin{array}{l}\text { Offre, produit, } \\
\text { organisation, } \\
\text { image, attractivité, } \\
\text { Diversité }\end{array}$} & \multirow{2}{*}{\multicolumn{2}{|c|}{$\begin{array}{l}\text { III-A. Aménagement } \\
\text { Équipement du lieu } \\
\\
\text { III-B. Usage/utilisation du lieu } \\
\text { Appropriation du lieu } \\
\text { Mode d'habiter/vivre } \\
\text { (touristiquement) du lieu } \\
\text { Pratique du lieu } \\
\text { Conflit d'usage } \\
\text { Partage du lieu } \\
\text { Fonction touristique/urbaine Pratique tour }\end{array}$}} & $\begin{array}{c}\text { Gestion } \\
\text { Développement } \\
\text { Planification, organisation } \\
\text { Politique } \\
\text { Projet/Projet de qualité }\end{array}$ & $\begin{array}{l}\text { Chose à voir, digne } \\
\text { d'un déplacement, } \\
\text { Destinée } \\
\text { Destin }\end{array}$ \\
\hline & & & & & $\begin{array}{l}\text { Concurrence/compét } \\
\text { (d'un véritable)/class } \\
\text { aspirer à devenir/s'in } \\
\text { identité/construction }\end{array}$ & $\begin{array}{l}\text { ition/rang } \\
\text { ement/qualification/ } \\
\text { qposer/qualité/image/ } \\
\text { de l'image, etc. }\end{array}$ \\
\hline & & & Fonction touristique & \multicolumn{2}{|c|}{ Fonction touristique/urbaine $\quad$ Activité (touristique/urbaine) } & \\
\hline $\begin{array}{l}\text { OPTIQUE GÉNÉRALE : } \\
\text { Extérieur/intérieur }\end{array}$ & À l'extérieur du lieu & $\begin{array}{l}\text { À l'extérieur et à } \\
\text { l'intérieur du lieu }\end{array}$ & \multicolumn{2}{|c|}{$\begin{array}{l}\text { À l'intérieur du lieu } \\
\text { (vision non intégrale) }\end{array}$} & $\begin{array}{l}\text { À l'intérieur du lieu } \\
\text { (vision intégrale) }\end{array}$ & \\
\hline $\begin{array}{l}\text { OPTIQUE DU POINT } \\
\text { DE VUE DE L'ACTEUR }\end{array}$ & $\begin{array}{l}\text { L'agent voyageant } \\
\text { (touriste) }\end{array}$ & $\begin{array}{l}\text { L'agent envoyant et } \\
\text { organisant le voyage } \\
\text { (voyagiste) et lieu } \\
\text { d'accueil }\end{array}$ & $\begin{array}{l}\text { III-A } \\
\text { Acteur accueillant: } \\
\text { aménageant le lieu }\end{array}$ & $\begin{array}{c}\text { III-B } \\
\text { Acteur accueillant et } \\
\text { voyageant qui utilise et } \\
\text { habite le lieu }\end{array}$ & $\begin{array}{l}\text { Acteur accueillant : planifiant, } \\
\text { développant, promouvant, } \\
\text { réalisant le projet du lieu } \\
\text { touristique selon une politique } \\
\text { et une stratégie }\end{array}$ & $\begin{array}{l}\text { L'agent voyageant et } \\
\text { lieu accueillant }\end{array}$ \\
\hline TYPE DE MOTIVATION & $\begin{array}{l}\text { Motivation du touriste } \\
\text { (but de déplacement) }\end{array}$ & $\begin{array}{l}\text { Motivation du touriste } \\
\text { et motivation du lieu } \\
\text { accueillant (attractivité) }\end{array}$ & \multicolumn{3}{|c|}{ Motivation = fonction du lieu } & $\begin{array}{l}\text { Motivation (du } \\
\text { touriste/du lieu) } \\
\text { comme nécessité } \\
\text { (destinée) }\end{array}$ \\
\hline $\begin{array}{l}\text { PHÉNOMĖNE NATUREL } \\
\text { vs CONSTRUIT }\end{array}$ & Phénomène naturel & $\begin{array}{l}\text { Phénomène naturel/ } \\
\text { construit }\end{array}$ & \multicolumn{3}{|c|}{$\begin{array}{l}\text { Phénomène construit } \\
\text { III-B : Phénomène naturel/construit }\end{array}$} & $\begin{array}{c}\text { Phénomène } \\
\text { construit qui se veut } \\
\text { naturel } \\
\end{array}$ \\
\hline $\begin{array}{l}\text { COMPATIBILITÉ AVEC } \\
\text { DES CLASSEMENTS }\end{array}$ & \multicolumn{2}{|c|}{$\begin{array}{l}\text { A : Type de ville touristique établi par caractère } \\
\text { géographique du lieu : destination touristique } \\
\text { urbaine vs destinations touristiques non urbaines } \\
\text { B1 : Classement des destinations (villes) selon } \\
\text { le type de motivation des touristes : destination } \\
\text { d'affaires, de jeu, d'accueil de congrès, etc. }\end{array}$} & \multicolumn{3}{|c|}{$\begin{array}{c}\text { C(1-2). Type du lieu — ville entière (C1)/zone, quartier (C2) — à l'égard } \\
\text { de la réalisation du projet/aménagement touristique : ville (zone) } \\
\text { de destination touristique vs ville (zone) qui n'a pas ce statut. } \\
\text { D. Type du milieu urbain touristique (localité entière) selon le type (degré) } \\
\text { de touristicité (motivation) du lieu : ville touristique vs ville touristifiée } \\
\text { vs station touristique, etc. } \\
\text { B2: Classement des destinations (villes entières/quartiers) selon le type de motiva- } \\
\text { tion (attractivité) dominante : destination d'affaires, de jeu, d'accueil de congrès, etc. } \\
\text { Classement des lieux selon le critère de la diversité : destination métropolitaine vs } \\
\text { d'autres types de la ville-destination }\end{array}$} & $\begin{array}{l}\text { F. Ville qui a réalisé } \\
\text { sa destinée } \\
\text { (potentielle) } \\
\text { touristique vs ville } \\
\text { qui n'y a pas réussi }\end{array}$ \\
\hline $\begin{array}{l}\text { RAPPORT } \\
\text { À LA DÉFINITION } \\
\text { DU TOURISME }\end{array}$ & $\begin{array}{l}\text { Tourisme }=\text { déplace- } \\
\text { ment + motivation } \\
\text { du déplacement } \\
\text { (d'agrémentrécréa- } \\
\text { tion/hors du quotidien) }\end{array}$ & $\begin{array}{l}\text { Tourisme }= \\
\text { déplacement }+ \\
\text { motivation du } \\
\text { déplacement }+ \\
\text { l'intermédiaire } \\
\text { dont les services } \\
\text { sont payants }\end{array}$ & $\begin{array}{r}\text { Tourisme }=\text { (déplac } \\
\text { utiliser et }\end{array}$ & $\begin{array}{l}\text { III-B } \\
\text { nent +) mode d'habiter et } \\
\text { jartager du lieu. }\end{array}$ & $\begin{array}{l}\text { Tourisme }=\text { une des fonctions } \\
\text { urbaines (quotidiennes); } \\
\text { sujet de la gestion et de la } \\
\text { politique (urbaines) } \\
\quad \downarrow \\
\text { L'urbanité est définie par sa } \\
\text { fonction touristique }\end{array}$ & $\begin{array}{c}\text { Tourisme }= \\
\text { accomplissement } \\
\text { d'un potentiel (devoir) } \\
\text { qui garantit le succès/ } \\
\text { l'adéquation à l'égard } \\
\text { des exigences de la } \\
\text { modernité/le futur. }\end{array}$ \\
\hline
\end{tabular}

Source : les auteurs. 
- la classification implicite selon le type de lieu (ville entière) à l'égard du statut de la réalisation du projet touristique $(\mathrm{Cl})$ : ville de destination touristique vs ville qui n'a pas ce statut;

- le classement explicite des types du milieu urbain touristique (localité entière) selon le type et le degré de la touristicité (motivation) du lieu (D) : ville touristique vs ville touristifiée vs station touristique, etc.;

- le classement implicite des lieux selon le critère de la diversité (E) : destination métropolitaine vs d'autres types de la ville-destination;

- le classement implicite selon le critère du succès du projet (F) : ville qui a réalisé sa destination (potentielle) touristique vs ville qui n'y a pas réussi.

En ce qui concerne les possibilités de définir le tourisme, l'acception IV, tout en mettant l'accent sur la gestion générale du lieu d'accueil en tant que milieu urbain, permet de définir le tourisme comme une des fonctions urbaines (quotidiennes). La variété III-A, qui considère la destination comme une zone mise en tourisme à travers l'aménagement et l'équipement appropriés, permet de définir le tourisme à travers le projet d'aménagement. Tandis que par association aux termes utiliser/approprier/ habiter/vivre/partager le lieu, la variété III-B permet de définir le tourisme comme mode d'habiter/utiliser et partager le lieu (urbain).

On observe que, dans toutes ses possibilités de définition, la composante «déplacement», bien que toujours présente, perd son importance. Par contre, la composante «motivation» subit une transformation : de la motivation du déplacement des touristes elle se développe vers la motivation (le but) de l'aménagement/projet de gestion ou se transforme en idée de fonction/utilisation du lieu l'accueil. Ce même mouvement vers l'idée de motivation s'observe au niveau des classements des destinations : le classement B2 est une variété inversée du classement B1, c'est-à-dire que la motivation du déplacement attribuée au touriste (B1) est réattribuée au lieu d'accueil (B2).

Rappelons que le champ sémantique usage/utilisation $d u$ lieu, appropriation (du lieu), habiter/vivre touristiquement (le lieu), fonction touristique, fonction urbaine, touristicité, urbanité constitue l'ensemble des contextes non favorables à l'apparition du concept destination. Ce lexique correspond à l'acception III-B (zone claire du tableau 5). Le paradoxe consiste en ce que la raison de cette incompatibilité ne paraît pas explicable à partir de la sémantique des termes en question. Les termes usage, utilisation, fonction ne font que reformuler l'aspect «emploi, usage» du terme destination. "L'incompatibilité» du mot destination avec ces termes pourrait servir de preuve indirecte de leur parenté sémantique avec le mot destination dans son acception «emploi»: leur synonymie aussi, bien que les structures syntaxiques différentes qu'ils impliquent ne favorisent pas leur croisement dans le même contexte.

Une énigme demeure : pourquoi les experts préferent-ils au mot destination ses synonymes? Ce paradoxe donne lieu à supposer que le terme destination est intuitivement perçu par les experts comme non approprié lorsque le contexte exige un concept sémantiquement simple et univoque pour décrire le fonctionnement du phénomène touristique et surtout pour chercher de nouveaux outils intellectuels pour le saisir (ce qui met fin à l'usage spontané). Voilà pourquoi le mot destination n'est pas trop bienvenu dans les textes qui parlent du déplacement (physique). Encore moins dans le contexte des termes usage/fonction/habiter/appropriation dont l'apparition dans le discours sur le tourisme marque le changement de l'approche méthodologique et par conséquent la révision de la conception de l'objet de recherche. De cette façon, avec le déplacement de la vision méthodologique vers l'idée de la ville chargée d'une fonction touristique vue comme l'usage du lieu, mode de vivre ou pratiquer $d u$ lieu, on entre dans une nouvelle zone épistémologique où le terme destination, impliqué dans un paradigme commun du discours scientifique en tourisme, s'avère peu approprié malgré ses capacités sémantiques.

\section{Destination comme direction de l'envoi organisé (variété II)}

Étant intégrée aux autres, l'acception II est difficile à trouver dans sa forme pure. Pour la distinguer de l'ensemble de variétés dominantes, il faut recourir à l'opposition mode actif $v$ s mode passif du déplacement : lieu où on se rend vs lieu où envoyer (cf. : «Point vers lequel on s'achemine ou on achemine un objet, une personne», Larousse, 1979). Le point de vue de l'acteur intermédiaire est ici mis en valeur. La particularité de cette acception est le mélange des optiques : «destination» est une direction du déplacement où l'on envoie des touristes, et en même temps un résultat de la production (lancement/fabrication/organisation) et de la gestion, un produit destinéa la vente, à la consommation et à la compétition. L'ajout sémantique de cette acception est donc «offre, produit de vente, attractivité et image promues».

Ce document est un outil pour tous les opérateurs publics ou privés s'occupant de la gestion de destinations urbaines (033-1877-2).

Si les [quatre] précédents types de pratique existent depuis l'invention du tourisme, un nouveau moteur du déplacement touristique et de la production des destinations est apparu au cours des deux dernières décennies (036-2056-1).

En définitive, la ville est une entité puissante, plurielle et hétérogène; de là vient sa complexité comme produit et destination touristique (039-2137-1).

Pour parler de la possibilité que l'acception II nous offre pour définir le tourisme, il faut mentionner des définitions fondées sur la formule déplacement + motivation et complétés par l'idée «d'intermédiaire» :

Le concept de pratique touristique renvoie à «la possibilité d'un arbitrage entre l'achat d'un produit touristique chez un intermédiaire et la volonté d'organiser soi-même son déplacement touristique» (036-2044-4).

\section{Destination comme destinée (variété $V$ )}

Dans l'entourage de l'ensemble des acceptions dominantes (II, III, IV), nous notons la présence forte des termes dont la signification comporte l'idée d'appréciation et de jugement de valeur. Parmi ces termes on retrouve espérer devenir, s'imposer comme destination, image, qualité, rang de (véritable) destination (recherchée), concurrence, compétition, etc. :

Ce formidable coup de projecteur va permettre à Marseille de se hisser à nouveau au rang de véritable destination touristique (051-2664-2). 


\section{LÉGENDE}

Inscriptions en blanc numérotées :

variétés sémantiques du terme destination

Inscriptions noires : termes-indices :

la cooccurrence avec eux caractérise la manifestation des variétés différentes du concept destination

\section{Sections foncées :}

contextes et variétés sémantiques du concept destination peu ou non favorables pour l'apparition du mot destination dans notre corpus

\section{Ligne blanche :}

montre la circulation du sens du concept destination, en revenant souvent à la variable de motivation (touristique).

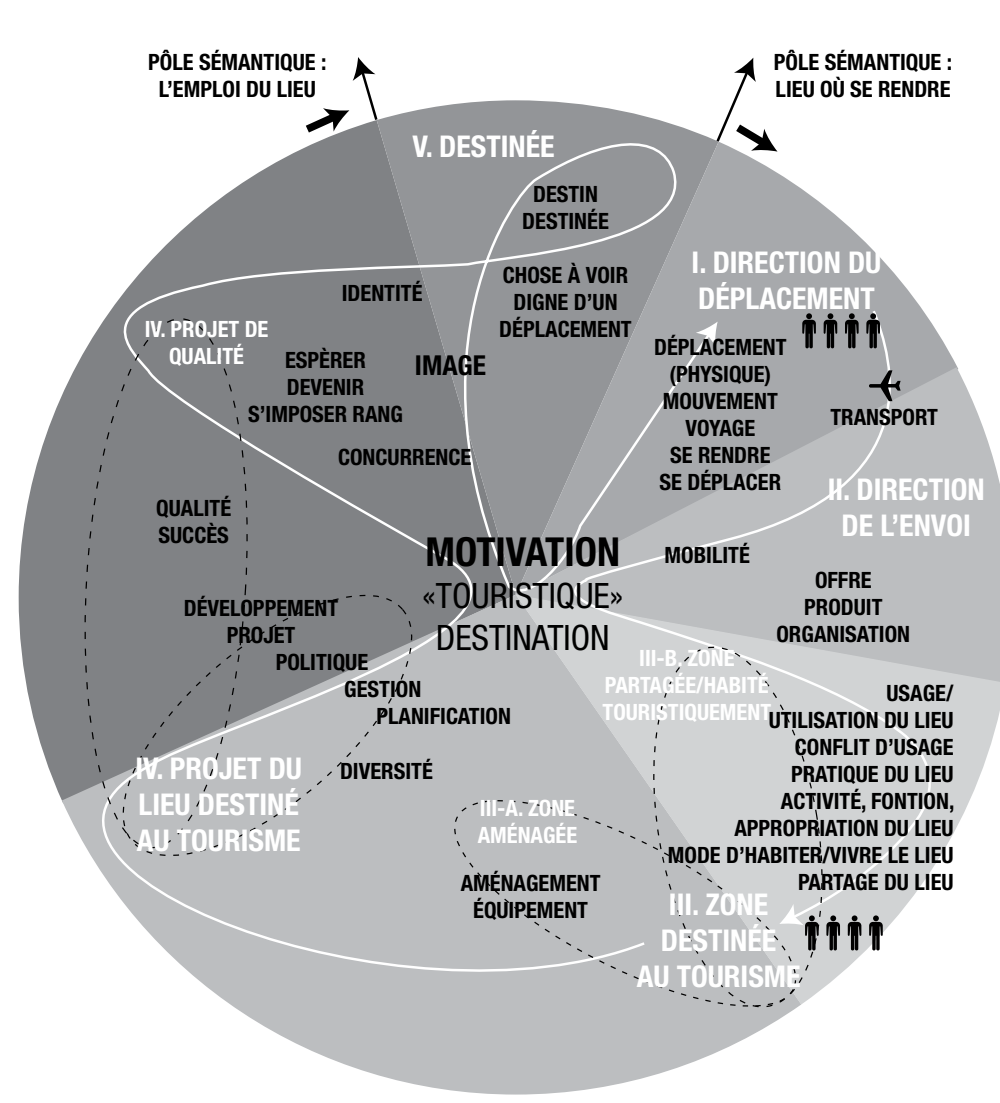

ILLUSTRATION 2 : Circulation du sens du concept de destination (source : réalisé par les auteurs à la lumière des résultats obtenus par Sémato).

Le tourisme peut aussi être lu comme une scène privilégiée sur laquelle les nouvelles destinations donnent à voir leur projet, parfois dans une compétition des monumentalités (036-2038-8).

Le facteur humain est une des clés du succès d'une destination touristique urbaine, c'est de la qualité des contacts dont le visiteur se souviendra (033-1926-1).

Le durcissement de la concurrence entre les villes les oblige à innover afin de rester au rang des destinations recherchées pour leur culture et de fidéliser leurs visiteurs (013-384-1).

Le statut de "destination» en tant que ville destinée au tourisme et aménagée d'une manière appropriée est potentiellement conçu comme un statut désiré, prestigieux, un garant de la réussite d'un futur assuré. Les classements $\mathrm{C}$ et $\mathrm{F}$ (voir tableau 4), implicitement présents dans le discours sur le tourisme, reflètent cette tendance.

Poussé à l'extrême, ce volet sémantique ouvre la possibilité d'une cinquième acception du terme destination, implicite, intégrée aux autres, mais bien perceptible. L'acception V met en évidence l'idée de devoir à accomplir, le choix prédestiné par la nécessité qui ne dépend pas de la volonté individuelle. Ainsi conçue, la destination réfère à la fois à la ville qui est destinée à accomplir son projet touristique et à la personne qui est impliquée dans un mode de vie où les activités touristiques sont dotées de valeur de nécessité ou de prestige. Voici quelques termes de même famille que destination récupérés dans notre corpus : destinée de la ville (1 occ.), destin touristique de la ville centre ( 2 occ.) aussi bien que chose à voir, digne d'un déplacement touristique (1 occ.).

Ainsi, il s'avère que la signification d'origine du terme destination («la fonction attribuée par une entité supérieure», «choix prédestiné de Dieu») abandonnée au début de notre analyse du fait de son caractère métaphysique revient toutefois s'installer au cour du discours scientifique en tourisme. Cette signification reste beaucoup plus visible dans le discours propre aux professionnels du tourisme et dans celui des publicitaires sur les destinations. Si l'on retire la composante métaphysique (Dieu), ce sont les représentations sociales et idéologiques formant et imposant un système des valeurs partagées dans une société donnée qui prennent alors place. On peut attester ici des traces d'une manipulation rhétorique décrite par Roland Barthes (1957) sous le nom de «naturalisation », c'est-à-dire la propriété des phénomènes d'ordre idéologique («mythologiques», selon Barthes) de se faire passer pour "naturels», propres à la nature des choses.

Selon l'acception V, le tourisme devrait se définir comme un accomplissement d'un potentiel (devoir) qui garantit le succès et l'adéquation à l'égard des exigences de la modernité et du futur. 


\section{Discussion-conclusion : L'hypothèse de la destination comme ensemble de "projets" différenciés mais intégrés à un "projet " global}

L'utilisation du terme destination dans le discours sur le tourisme (urbain) n'est ni transparente ni univoque. L'ambigüité inhérente peut être reconnue comme une caractéristique principale du fonctionnement du terme en question. Cette ambigüité, qui augmente au fur et à mesure de l'éloignement de l'idée pure du déplacement physique, est due à la polysémie interne du terme destination aussi bien qu'à l'ambigüité sémantique de son encadrement, lexical et syntaxique (Les résultats de l'analyse des facteurs syntaxiques permettant de comprendre le mécanisme linguistique contribuant à l'ambigüité sémantique du terme destination n'ont pas été inclus dans le présent article. Ils feront l'objet d'une publication ultérieure.). Jamais définie, la signification du concept se déplace continuellement et se modifie, souvent jusqu'à devenir méconnaissable, en fonction du changement du point de vue des experts sur leur objet d'études.

Dans le discours sur le tourisme, contrairement à l'usage normal, les variétés différentes du concept destination ne sont pas clairement distinguables. Les contextes favorisant telle ou telle acception se chevauchent et passent de l'un à l'autre : l'illustration 2 cherche à expliciter la circulation fluide du sens du terme destination organisée autour de la variable motivation dont le représentant majeur est le terme touristique.

Par ailleurs, le terme destination s'avère sélectif à l'égard des contextes qui paraissent bien compatibles avec lui. Ainsi, il n'est pas utilisable avec des concepts du champ usage/habiter/fonction, etc. Il est aussi peu utilisable dans le contexte du déplacement purement physique. En revanche, le terme destination est utile quand on cherche à saisir l'objet d'étude dans sa complexité à l'échelle globale (peu importe s'il s'agit de la vision de l'intérieur ou de l'extérieur de la ville). Il ne serait probablement pas erroné de dire que les limites de la puissance et l'intérêt d'utilisation du concept de destination dans le discours sur le tourisme coïncident avec les limites de son ambigüité sémantique. Devrions-nous conclure que le comportement du concept de destination témoigne de son déficit sémantique et par conséquent de son inaptitude à servir de bon terme pour le discours scientifique en tourisme? Cette question provocatrice nous incite à poursuivre la réflexion, mais nous amène toutefois à relever plusieurs observations.

- La complexité au niveau du langage reflète sans doute la nature complexe du phénomène du tourisme (urbain) lui-même. L'essentiel de ce phénomène émerge au croisement de plusieurs facettes. Il est logique de supposer que ces facettes impliquent différentes "optiques» liées à différentes définitions et classifications. C'est de là que vient le besoin de termes adéquats qui seront capables de renfermer plusieurs optiques à la fois. Le terme destination s'avère être assez utile pour combler ce besoin et refléter la complexité essentielle de l'objet d'étude. Pourtant, il est clair que le terme destination dans le discours sur le tourisme reste un terme cognitif : une empreinte au langage quotidien auquel la définition (les définitions) a été accordée intuitivement, sans mise à l'épreuve d'une réflexion métalinguistique systématique.
- Le problème soulevé par l'analyse sémantique du concept destination s'avère plus profond qu'il n'a paru au début. Comme nous l'avons vu, l'ambigüité terminologique fondée sur la confusion des repérages spatiaux et des optiques des acteurs ne constitue pas une caractéristique exclusive du terme destination. Elle se manifeste à différents niveaux du discours en tourisme. Une analyse sémantique en cours portant sur d'autres termes tel que «tourisme urbain» prouve l'hypothèse de l'insuffisance terminologique auquel fait face la construction du discours scientifique en tourisme.

- L'analyse sémantique du concept de destination démontre que l'intérêt de l'utilisation du terme destination dans le discours sur le tourisme est associé de façon probante avec l'idée de projet. Si l'on comprend par «projet» des activités intentionnelles et intéressées projetées dans le futur et dont le but est de changer l'état actuel des choses (selon son étymologie, le mot projet veut dire "jeter quelque chose vers l'avant»), cet aspect sémantique est bien présent dans l'ensemble des termes qui favorisent, dans notre corpus, l'apparition du mot destination : gestion, développement, planification, politique, etc.

Cette dernière note nous conduit à proposer une définition du concept de destination en tant que terme fondamental de la science touristique à travers le développement de son rapport avec le concept de projet. L'idée de "projet» associée à "destination" a été avancée par certains auteurs, dont Knafou et Stock (2003), Ceriani et al. (2008) et Violier (2009), où l'on observe que le projet est une construction du touriste. Or, et malgré l'effort de dépassement des lectures unidimensionnelles et réductrices du concept de destination, le projet n'identifie qu'une composante de la globalité de la destination. Nous pensons qu'il est nécessaire de parler de la destination en termes de "projets", au pluriel et non au singulier comme le font les auteurs mentionnés. Notre hypothèse est que la destination se présente comme un ensemble dynamique de projets conçus comme efforts intentionnels et intéressés des acteurs intervenant dans la construction de la destination. Ces projets, à la fois différenciés mais concourant à la formation d'un projet intégré, renvoient à des dimensions diverses. Ainsi, nous distinguerions

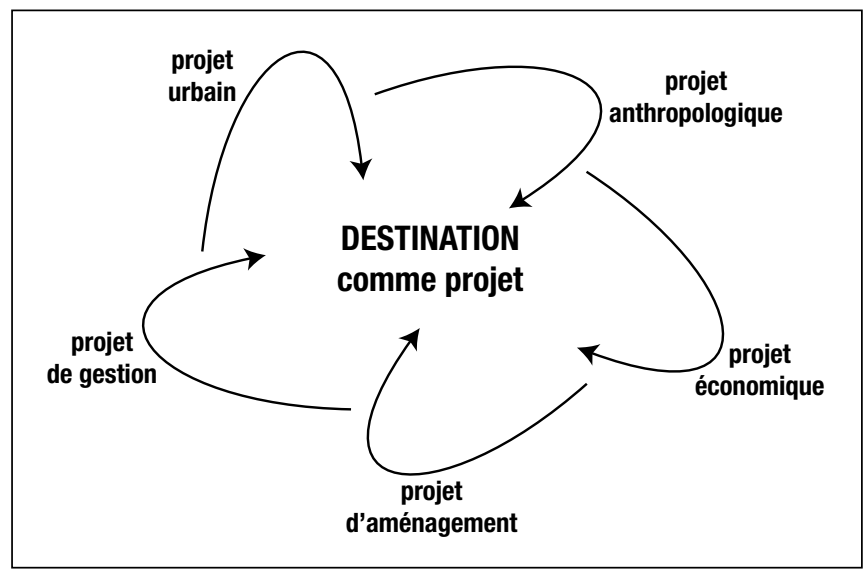

ILLUSTRATION 3 : Modèle de la destination comme ensemble de projets (source : les auteurs). 
cinq projets liés organiquement entre eux : un projet anthropologique (vision des leaders, motivation des touristes), un projet économique (marché, produit touristique), un projet d'aménagement (mise en tourisme des espaces), un projet de gestion (mécanisme d'organisation et de gouvernance), un projet urbain (projet sociétal) (voir illustration 3 ).

Cette perspective contribuerait au renouvellement de la compréhension de la destination en tant que concept et projet de société dans le cadre de la destination urbaine, en s'enrichissant notamment d'une approche transdisciplinaire. En faisant intervenir certains modèles de la théorie des organisations (différentiation-intégration et modèle des intéressés), des études urbaines (le concept de projet urbain), de la psychologie (le concept de résilience appliqué à la ville), nous pourrions envisager la compréhension et l'évaluation de la destination autrement que par le modèle de cycle de vie qui ne rend pas compte de la riche complexité de cette dernière.

\section{Note}

1 Les citations données dans cette rubrique sont toutes extraites du corpus. L'objet d'étude étant la particularité du discours général en tourisme plutôt que les opinions de tels ou tels experts, nous trouvons possible de ne pas donner de références à des sources publiées et de conserver l'encodage de l'analyse statistique. Le premier nombre indique le numéro du document dans le corpus, le deuxième le numéro du paragraphe, le troisième le numéro de la phrase dans le paragraphe.

\section{Références}

BARTHES, Roland (1957) Mythologies, Paris : Seuil. 267 p.

BLOOMFIELD, Leonard (1933, r. 1961) Language, New York : Holt, Rinehart \& Winston. 566 p.

BOTTI, Laurent; Nicolas PEYPOCH et Bernardin SOLONANDRASANA (2008) «De la destination touristique», chapitre 1 DANS Ingénierie du tourisme: Concepts, Méthode, Applications, p. 19-33. Bruxelles : De Boeck, coll. «les Métiers du tourisme».

BRUNET, Roger (1993) Les mots de la géographie. Dictionnaire critique, Paris : La Documentation française, coll. «Dynamiques du territoire», $518 \mathrm{p}$.

BUTLER, Richard (1980) "The concept of a tourist area cycle of evolution : implications for management of resources", The Canadian Geographer, $\mathrm{n}^{\circ} 24$, vol 1, p. 5-12.

Centre national de ressources textuelles et lexicales (CNRTL) (2009) Dictionnaire étymologique en ligne, <http://www.cnrtl.fr/etymologie/>, consulté en mars 2010.

CERIANI, Giorgia; Vincent COËFFÉ; Jean Christophe GAY; Rémy KNAFOU; Mathis STOCK et Philippe VIOLIER (2008) Conditions géographiques de l'individu contemporain, Espace temps. net, <http://www.espacestemps.net/document4573.html>, consulté le 20 novembre 2009.

CINOTTI, Yves (2009) «L'hospitalité touristique au service des destinations ", dans LEMASSON, Jean Pierre et Philippe VIOLIER (sous la direction de), Destinations et territoires, p. 92-100. Sainte-Foy : Éditions Téoros, Presses de l'Université du Québec.

DAVIDSON, Rob et Robert MAITLAND (2002) Tourism destinations, the dynamics and evolution of tourism destination, London : Hodder and Stoughton. $281 \mathrm{p}$.

DUCROT, Oswald et Jean-Marie SCHAEFFER (1999) Nouveau dictionnaire encyclopédique des sciences du langage, Paris : Seuil. $817 \mathrm{p}$.
ETCHNER, Charlotte et Brent RITCHIE (1991) "The Meaning and measurment of destination image", The Journal tourism studies, $\mathrm{n}^{\circ}$ 2, p. 2-12.

Équipe MIT (2002) Tourisme 1, Paris : Belin. 320 p.

Équipe MIT (2005) Tourisme 2, Paris : Belin. 349 p.

GALLARZA, Martina; Irene Gil SAURA et Haydée Calderon GARCIA (2002)

"Destination image : Towards a Conceptual Framework", Annals of tourism research, vol. 29, $\mathrm{n}^{\circ}$ 1, p. 56-78.

GIBSON, Alex et Michel TIARD (1999) «Le marketing de la destination touristique : management de la destination et gestion de la marque», Cahier espaces, $\mathrm{n}^{\circ}$ 64, p. 76-87.

GOELDNER, Charles et J. R. Brent RITCHIE (2006) Tourism : principles, practices, philosophies, $10^{e}$ édition, Hoboken (NJ) : John Wiley \& Sons. 521 p.

HAYLLAR, Bruce; Tonny Griffin et Deborah Edwards (2008) City spaces, Tourist places. Urban tourism precincts. Amsterdam : Butterworth-Heinemann, Elseiver. $381 \mathrm{p}$.

HAZEBROUCQ, Jean-Marie (2009) «De l'espace touristique à la destination touristique : un territoire qui se projette», dans LEMASSON, Jean Pierre et Philippe VIOLIER (sous la direction de), Destinations et territoires, p. 7-27. Sainte-Foy : Éditions Téoros, Presses de l'Université du Québec.

JAFARI, Jafar (2000) Encyclopedia of tourism, p. 144-145. London : Routledge.

KADRI, Boualem (2008) «Lidentité scientifique du tourisme : un mythe ou une réalité en construction", Revue de recherche en tourisme : Téoros, vol 27, $\mathrm{n}^{\circ} 1$, printemps, p. 51-58.

KEROURIO (2007) Géotourisme: Le site de la Géographie touristique en France et dans le Monde, Dicotour, <http://geotourweb.com/>, consulté en mars 2010.

KNAFOU, Rémy et Mathis STOCK (2003) «Tourisme», dans LÉVY, Jacques et Michel LUSSAULT (dir.), Dictionnaire de la géographie et de l'espace des sociétés, p. 931-933. Paris : Belin.

KYE-SUNG, Chon (1990) "The rôle of destination image in tourism : A review and discussion", Revue du tourisme, $\mathrm{n}^{\circ}$ 2, p. 2-9.

LAROUSSE (1979) Le Lexis, Dictionnaire de la langue française, Larousse, 2109 p. LESNE, Anaïs et Michel ZINS (2009) «Évaluer les destinations touristiques dans un contexte de multiplication des expériences offertes aux touristes internationaux», DANS LEMASSON Jean Pierre et Philippe VIOLIER (sous la direction de), Destinations et territoires, p. 23-37. Sainte-Foy : Éditions Téoros, Presses de l'Université du Québec.

LEVY, Jacques et Michel LUSSAULT (dir.) (2003) Dictionnaire de la géographie et de l'espace des sociétés, p. 931-934. Paris : Belin.

OLIVIER, Jean-Jacques (2006) Le Dicotour : dictionnaire pratique des 2000 mots et sigles du tourisme, Paris : Éditions Technip. 152 p.

ONFRAY, Michel (2007) Théorie du voyage : poétique de la géographie, Paris : Éditions Livre de Poche. 126 p.

REY, Alain (dir.) (1992) Dictionnaire historique de la langue française, Paris : Dictionnaires Le Robert. 4304 p.

STABLER, Mike (1988) "The image of destination regions : theoretical and empirical aspects”, DANS GOODALL, B. et G. ASHWORTH, Marketing in tourism industry: The promotion of destination régions, p. 133-161. Londres : Édition Croom Helm.

TELISMAN-KOSUTA, Neda (1989) Tourism destination image, DANS WITT, S. F. et L. Moutinho (dir.), Tourism marketing and management handbook, p. 557-561. Cambridge : Édition Prentice Hall.

TOCQUER, Gérard et Michel ZINS (1999) Marketing du tourisme, deuxième édition, Paris : Gaëtan Morin. 303 p.

VIOLIER, Philippe (2009) «Proposition pour un modèle d'analyse et de management des lieux touristiques», DANS LEMASSON, Jean Pierre et Philippe VIOLIER (sous la direction de), Destinations et territoires, p. 23-37. Sainte-Foy : Éditions Téoros, Presses de l'Université du Québec. 